\title{
Reconstrução laringotraqueal em único estágio para tratamento de estenose subglótica em crianças
}

\section{Single-stage laryngotracheal reconstruction for the treatment of subglottic stenosis in children}

\author{
Mariana Magnus Smith ${ }^{1}$, Cláudia Schweiger ${ }^{2}$, Denise Manica ${ }^{3}$, Camila Degen Meotti ${ }^{4}$, Larissa Valency Eneas ${ }^{5}$, Gabriel Kuhl', \\ Paulo Jose Cauduro Marostica?.
}

1) Mestre. Preceptora da Residência Médica em Otorrinolaringologia do Hospital São Lucas da PUCRS.

2) Mestre. Preceptora da Residência Médica em Otorrinolaringologia do Hospital de Clínicas de Porto Alegre

3) Otorrinolaringologista. Fellowship em Laringologia do Hospital de Clínicas de Porto Alegre.

4) Otorrinolaringologista. Fellowship em Rinologia do Hospital de Clínicas de Porto Alegre.

5) Otorrinolaringologista. Preceptora da Residência Médica em Otorrinolaringologia do Hospital São Lucas da PUCRS.

6) Otorrinolaringologista. Professor do Departamento de Otorrinolaringologia e Oftalmologia da UFRGS. Preceptor da Residência Medica em Otorrinolaringologia do HCPA.

7) Pós-doutorado. Professor do Departamento de Pediatria da UFRGS. Preceptor da Residência Medica de Pediatria do HCPA.

Instituição: Hospital de Clínicas de Porto Alegre.

Porto Alegre / RS - Brasil.

Endereço para correspondência: Cláudia SchweigerRua Ramiro Barcelos, 2350 - Zona 19 - Porto Alegre / RS - Brasil - CEP: $90035-903$ - E-mail: causch@ hotmail.com Artigo recebido em 7 de Setembro de 2011. Artigo aprovado em 12 de Outubro de 2011.

\section{RESUMO}

Introdução: Nas últimas décadas, a reconstrução da via aérea tornou-se o tratamento de escolha para estenose subglótica (ESG) na criança, realizada em único ou múltiplos estágios. Há indícios na literatura de que a cirurgia em um só tempo é mais efetiva.

Objetivo: Avaliar o índice de sucesso da laringotraqueoplastia (LTP) e ressecção cricotraqueal (RCT) em único estágio nos pacientes tratados no nosso hospital.

Método: Estudo retrospectivo de crianças submetidas à reconstrução laringotraqueal.

Resultados: Foram incluídos 24 pacientes. As causas da ESG foram pós-intubação endotraqueal em 91,6\% e congênita em 8,3\%. Diagnosticamos ESG grau 4 em 1 paciente (4\%), grau 3 em 16 (66,6\%), grau 2 em 4 (16,6\%), grau 3 associada com estenose glótica em 1 (4\%), grau 3 associada com estenose traqueal em 1 (4\%). Foram realizadas 26 LTP e 3 RCT. O índice de decanulação foi de $66 \%$ nos pacientes submetidos à RCT e de $85,7 \%$ nos pacientes submetidos à LTP; o índice total de decanulação foi de 83,3\%. Todos apresentaram febre persistente no pós-operatório, que cessou após a extubação.

Conclusão: Nossa série mostrou índice de decanulação de 83,3\%.

Palavras-chave: laringe, criança, laringoestenose.

\section{SUMMARY}

Introduction: In recent decades, airway reconstruction has become the treatment of choice for subglottic stenosis (SGS) in children, which is performed in either single or multiple stages. However, there is evidence in the literature that singlestage surgery is more effective.

Objective: To evaluate the success rate of single-stage laryngotracheoplasty (LTP) and cricotracheal resection (CTR) in patients that were treated in our hospital. Materials and Method: We performed a retrospective study of children undergoing laryngotracheal reconstruction.

Results: Twenty-four children were included. The etiology of SGS was postintubation in $91.6 \%$ and congenital in $8.3 \%$. One patient (4.2\%) had grade 4 SGS, $17(70.8 \%)$ presented with grade 3 SGS, 4 (16.6\%) had grade 2 SGS, 1 (4.2\%) had grade 3 SGS associated with glottic stenosis, and 1 (4.2\%) had grade 3 SGS with tracheal stenosis. We performed 26 LTPs and 3 CTRs. Decannulation rates were 66\% in the CTR procedures and $85.7 \%$ in the LTP procedures; the overall decannulation rate was $83.3 \%$. All children presented with fever in the postoperative period, but were afebrile after the tube was removed.

Conclusion: Our series showed a decannulation rate of $83.3 \%$. Keywords: larynx, child, laryngostenosis. 


\section{INTRODUÇÃO}

Nas últimas três décadas, a reconstrução da via aérea, tanto através de laringotraqueoplastia (LTP) (aumento do diâmetro da via aérea através de enxertos de cartilagem) quanto através da ressecção cricotraqueal (RCT) (exérese da cartilagem cricoide e/ou de anéis traqueais estenosados e anastomose término-terminal), tornou-se o tratamento de escolha para estenose subglótica (ESG) na criança.

A LTP foi descrita pela primeira vez em 1974 por Evans e Tood (1), mas ganhou popularidade com Cotton no final dos anos 70 , com a introdução do uso de enxerto de cartilagem costal (2). A RCT é utilizada mais comumente em crianças com estenoses graves (graus III e IV MyerCotton) (3).

A reconstrução laringotraqueal pode ser realizada em um único estágio, em que o paciente permanece com um tubo endotraqueal (TET) por um determinado período no pós-operatório, ou em múltiplos estágios, em que o paciente permanece com traqueostomia por tempo indeterminado no pós-operatório. Há indícios na literatura de que a cirurgia em um só tempo é mais efetiva do que a realizada em dois tempos, pois o TET já funcionaria como molde da via aérea no pós-operatório, servindo como stent temporário e evitando granulação obstrutiva $(10,11)$.

Desse modo, a realização da cirurgia em um só tempo parece ser uma tendência mundial, e em nosso Serviço optamos sempre pela sua realização em casos de ESG com indicação de cirurgia aberta.

Assim, nosso objetivo é avaliar o índice de sucesso da LTP e RCT em único estágio nos pacientes pediátricos realizadas pelo Serviço de Otorrinolaringologia do nosso Hospital, comparando-o com os índices relatados na literatura, além de descrever as características destes pacientes.

\section{Método}

Realizamos um estudo retrospectivo através da revisão dos prontuários de todos os pacientes do nosso Serviço submetidos à reconstrução laringotraqueal no período de junho de 2005 a novembro de 2009. Foram incluídos os pacientes pediátricos com estenose laríngea e/ ou traqueal proximal submetidos LTP e RCT em um único estágio.

O projeto foi aprovado pelo Comitê de Ética em Pesquisa do nosso Hospital, sob o número 09-568.
Os dados foram analisados no programa PASW 17.0.

A seguir, descreveremos os procedimentos com detalhes.

\section{LTP em unico estágio}

Se o paciente não estiver traqueostomizado, realizase a intubação endotraqueal. Caso contrário, a ventilação do paciente durante a cirurgia se faz através da cânula de traqueostomia.

Posiciona-se o paciente em hiperextensão cervical. Infiltram-se os locais da cirurgia (região cervical anterior e região torácica doadora da cartilagem costal) com lidocaína 1\% e adrenalina 1:100.000. O tórax é abordado em primeiro lugar, minimizando o risco de contaminação; por convenção, o enxerto é retirado na região submamária direita adjacente à junção ósteo-cartilaginosa, tendo-se o cuidado para removê-lo em um plano subpericondreal (segmento com 2 a 3 cm de extensão).

A seguir, é realizada incisão horizontal cervical e um retalho subplatismal é confeccionado, com identificação das cartilagens cricoide e tireoide, bem como da traqueia. Após, procede-se à laringofissura anterior na linha média, e, se indicada (necessidade de colocação de enxerto posterior na cricoide), é também realizada abertura da parede posterior da cartilagem. Nesta hora, se o paciente estiver intubado por via alta, troca-se o tubo endotraqueal para abaixo da área estenótica. Aárea estenosada é medida e o enxerto em formato fusiforme é preparado (Figura 1). O enxerto é posicionado, primeiramente, na parede posterior, e com o pericôndrio voltado para a porção interna da via aérea, sendo suturado com PDS 5.0 (Figura 2). É introduzido um tubo nasotraqueal, servindo como molde da via aérea, e se realiza a colocação do enxerto anterior (se necessário), com fechamento de toda a via aérea, suturandose inclusive o local da traqueostomia prévia, se presente. Posiciona-se dreno de Penrose e realizam-se as suturas por planos.

\section{RCT}

Geralmente há traqueostomia prévia (ESG Grau 4) e o tubo endotraqueal é passado através do estoma. Posiciona-se o paciente em hiperextensão cervical. A infiltração é realizada somente na região cervical. Realizase incisão elíptica ao redor do traqueostoma e pode-se elevar um retalho subplatismal superiormente até o nível do osso hioide e inferiormente até a fúrcula esternal (dependendo do local e da extensão da estenose). A via aérea é aberta com uma incisão vertical na linha média 


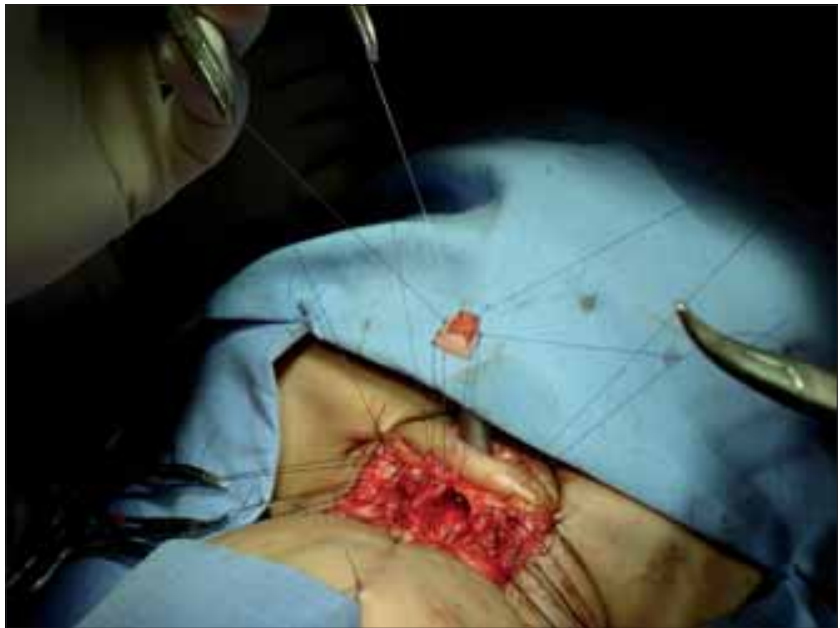

Figura 1. Fios de sutura colocados no enxerto de cartilagem costal e na parede posterior da cricoide (laringofissura posterior), antes do posicionamento definitivo do enxerto. Nota-se o tubo endotraqueal colocado no traqueostoma, abaixo da área de estenose.

através da cartilagem cricoide e a estenose é examinada. Define-se a margem superior da ressecção de acordo com a estenose (por exemplo, se for necessário retirar parte da cricoide, essa ressecção deve estender-se lateralmente pela borda inferior da cartilagem tireoide em direção à articulação cricotireoidea). É realizada a mobilização da traqueia, sempre bem próximo à parede traqueal no plano subpericondreal, e a mesma é elevada com auxílio de duas suturas traqueais laterais com prolene 2.0. A margem de ressecção inferior deve conter um anel traqueal completamente sadio. É desfeita e hiperextensão cervical e posiciona-se um tubo nasotraqueal, realizandose as suturas da anastomose término-terminal com PDS 2.0. Posiciona-se dreno Penrose e realiza-se o fechamento por planos.

Tanto na LTP quanto na RCT, o paciente é encaminhado à Unidade de Terapia Intensiva Pediátrica no pósoperatório, onde permanecerá intubado e sedado. No sétimo dia pós-operatório, realiza-se laringoscopia direta com óptica, retirando-se o TET para a visualização de toda a via aérea. Se a área operada estiver pérvia, sem tecido de granulação abundante e com os enxertos bem posicionados (LTP), reintuba-se o paciente sob visualização direta e iniciam-se a diminuição da sedação e o processo de extubação. No sétimo dia após a extubação, realiza-se uma nova laringoscopia direta e, estando a via aérea pérvia e o paciente assintomático, libera-se o paciente para casa e agenda-se retorno ambulatorial para acompanhamento. Se necessário, utilizamos terapias complementares, como laser de $\mathrm{CO}_{2}$, dilatações ou nova intubação e/ou traqueostomia.

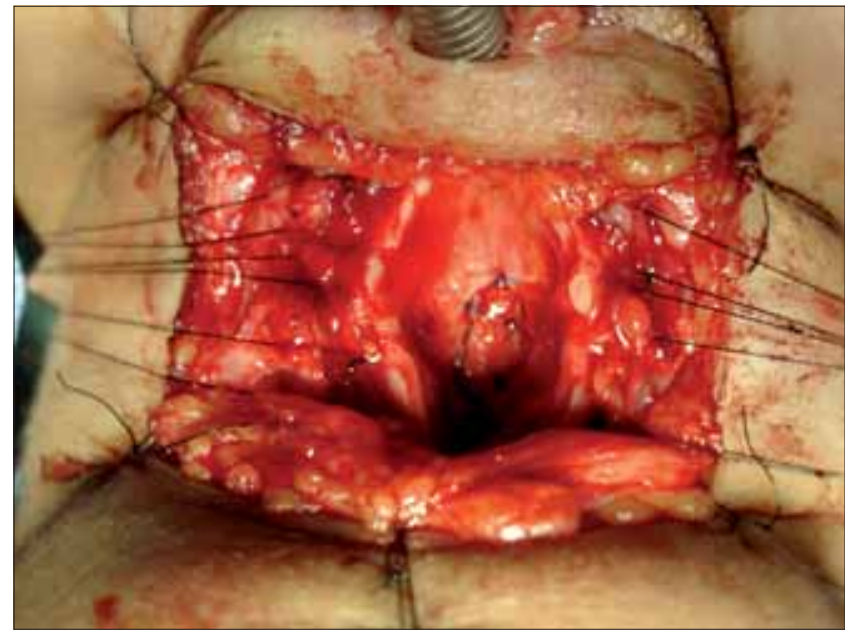

Figura 2. Laringofissura posterior com visualização do enxerto de cartilagem costal suturado com PDS 5.0. Notam-se os fios de mononylon para afastamento das paredes da cricoide e traqueia durante a cirurgia, dispensando auxiliares e a colocação de afastadores que possam lesar estas estruturas.

\section{RESULTADOS}

Foram revisados os prontuários de 24 pacientes, com idades entre 2 meses e 17 anos, submetidos à LTP ou RCT para correção de estenose subglótica e/ou traqueal.

\section{Características da população}

Treze pacientes (54\%) eram do sexo masculino.

Dos 24 pacientes, 17 (71\%) não apresentavam comorbidades. As comorbidades encontradas nos demais foram: prematuridade (3), epilepsia (1), síndrome de Fraser (1), Síndrome de Down (1) e atresia de via biliar(1).

As causas da ESG foram intubação endotraqueal em 22 pacientes $(91,6 \%)$ e congênita em 2 pacientes $(8,3 \%)$.

Dos pacientes que tiveram como causa da estenose a intubação endotraqueal, a média de dias de intubação foi de 11,7 dias, variando de 5 a 28 dias. Em relação à causa da intubação, encontramos: bronquiolite em 7 (31,8\%), pneumonia em 2 (9\%), coqueluche em 2 (9\%), trauma em $3(13,6 \%)$, encefalite viral em 1 (4,5\%), sepse neonatal em $1(4,5 \%)$, transtornos relacionados à prematuridade em 2 (9\%), aspiração de mecônio em 1 (4,5\%), traqueomalacia em $1(4,5 \%)$, tuberculose pulmonar em 1 $(4,5 \%)$ e complicações em pós-operatórios de outras cirurgias em $1(4,5 \%)$. 
No diagnóstico pré-operatório, a classificação da estenose encontrada foi a seguinte: ESG grau 4 em 1 paciente (4\%), ESG grau 3 em 16 pacientes (66,6\%), ESG grau 2 em 4 pacientes (16,6\%), ESG grau 3 associada com estenose glótica em 1 paciente (4\%), ESG grau 3 associada com estenose traqueal em 1 paciente (4\%).

Antes da cirurgia definitiva, 18 pacientes (75\%) necessitaram traqueostomia por disfunção respiratória.

Não considerando a traqueostomia, a LTP foi o primeiro tratamento instituído em 19 pacientes (79,1\%), a RCT foi o primeiro tratamento em 3 pacientes $(12,5 \%)$ e a utilização de laser de $\mathrm{CO}_{2}$ foi o tratamento de escolha inicial em 2 pacientes $(8,3 \%)$.

Foram realizadas 26 LTP (21 primárias e 5 reintervenções). Em relação aos enxertos, foram colocados enxertos posteriores em 9 cirurgias (34,6\%), enxerto anteriores em 2 cirurgias $(7,7 \%)$ e enxertos associados (anterior e posterior) em 15 cirurgias $(57,7 \%)$.

Não foram relatadas intercorrências no transoperatório. As complicações pós-operatórias observadas foram: 2 pneumonias, 1 trombose de veia jugular interna, 1 deslocamento do enxerto e 1 óbito por choque séptico. O paciente que apresentou deslocamento do enxerto sofreu reintervenção no 14 ํ pós-operatório, 7 dias após a extubação da primeira cirurgia.

O período médio de intubação pós-operatória foi de 7,7 dias.

Todos os pacientes apresentaram febre persistente enquanto permaneceram na Unidade de Terapia Intensiva. Após a extubação, a febre cessou em todos eles. Foi realizada investigação laboratorial da febre, mas causa infecciosa foi elucidada somente em 3 casos: 2 pacientes com pneumonia e 1 com choque séptico (e óbito).

\section{Taxas de sucesso}

Entre os 24 pacientes operados, 15 (62,5\%) apresentaram via aérea adequada (sem estenose (12 pacientes) ou com ESG grau 1 assintomática (3 pacientes)) após a primeira cirurgia de reconstrução da via aérea, sendo decanulados e não apresentando necessidade de reintervenções posteriores.

Cinco pacientes foram submetidos a reintervenções (nova LTP, RCT, laser de $\mathrm{CO}_{2}$ ou dilatação com balão), apresentando luz subglótica adequada e decanulação após esse segundo procedimento.
Três pacientes foram submetidos a traqueostomia após a primeira cirurgia por reestenose. A reintervenção ainda não foi realizada.

Uma paciente foi a óbito no pós-operatório por choque séptico, sendo considerada como insucesso para a análise dos casos.

Todos as laringoscopias diretas realizadas na Unidade de Terapia Intensiva no sétimo dia pós-operatório mostraram via aérea pérvia, com pouca ou nenhuma quantidade de tecido de granulação, permitindo a extubação dos pacientes logo após.

O índice de decanulação foi de 66\% nos pacientes submetidos à RCT e de $85,7 \%$ nos pacientes submetidos à LTP. O índice de decanulação total foi de 83,3\%.

\section{DISCUSSÃO}

O manejo da estenose laringotraqueal é um desafio para os otorrinolaringologistas, especialmente na população pediátrica. Muitas vezes são casos complexos, em que temos de utilizar várias modalidades de tratamento para a completa resolução do quadro. Devem ser levados em consideração o tipo de estenose, localização, extensão, grau de obstrução da via aérea, comprometimento das pregas vocais e as alterações neurológicas do paciente

Existem diversos tipos de intervenções cirúrgicas para o manejo da estenose: cirurgia endoscópica com laser associada ou não com dilatação ou stents; dilatação com balão de angioplastia; LTP com enxerto de cartilagem costal anterior, posterior ou combinação de ambos realizados em único ou múltiplos estágios; e a RCT. No presente estudo, descrevemos os resultados cirúrgicos do nosso serviço para o tratamento de estenose subglótica e traqueal, onde foram utilizadas as técnicas de LTP e RCT em único estágio, utilizando o TET como molde para a via aérea no pós-operatório.

Em relaçãoà causa da estenose, a intubação traqueal foi relacionada a $91,4 \%$ dos pacientes, concordando com estudos prévios que relatam que $90 \%$ das estenoses subglóticas adquiridas têm história de intubação (4); e 8,6\% dos pacientes apresentavam estenose congênita, cuja incidência na literatura é em torno de 5\% (5).

Relatos de alguns centros têm mostrado séries de LTP em único estágio com taxas de decanulação variando de $84 \%$ a 96\% (6). Nos casos de RCT, as taxas de decanulação variam de $91 \%$ a 95\% (7). Em nossa série, o índice de decanulação geral foi de $83,3 \%$. Quando analisados separadamente, os pacientes submetidos a RCT apre- 
sentaram taxa de decanulação de 66\%. Provavelmente essa taxa mais baixa do que a relatada na literatura devase ao pequeno número de pacientes submetidos a RCT na nossa série. É importante ressaltar que a RCT era considerada até o fim dos anos noventa uma abordagem quase que exclusiva de adultos (8), sendo, portanto, uma cirurgia relativamente nova na população pediátrica. Quando analisada apenas a LTP, a taxa de decanulação foi de $85,7 \%$, compatível com a relatada por outros autores.

Até há cerca de um ano não utilizávamos a dilatação com balão (cateter de angioplastia) no pós-operatório dos pacientes submetidos a reconstrução laringotraqueal, mas, no momento em que passamos a utilizar este dispositivo, notamos uma maior facilidade no manejo do tecido de granulação subglótico destes pacientes. A laringoplastia com balão pode vir a modificar nossas taxas de sucesso pós-reconstrução laringotraqueal, pois os quatro casos que apresentaram reestenose poderiam ter sido submetidos a dilatação no pós-operatório, no momento em que ainda apresentavam tecido de granulação, o que poderia ter mudado a sua evolução.

Todas as laringoscopias diretas realizadas no sétimodia pós-operatório mostraram via aérea adequada e permitirama extubação dos pacientes, o que tem nos levado a reconsiderar a real necessidade deste exame antes da extubação.

A febre pós-operatória parece ser comum após a reconstrução da via aérea. Estudo realizado por Schraff et al, em 2010, mostrou que 59\% dos casos de LTP em único estágio apresentaram febre no pós-operatório; porém, ela foi considerada significativa (com culturais positivos) em apenas um terço dos casos (9). Na amostra estudada, todos os pacientes apresentaram febre no pós-operatório, a qual cessou após a remoção do TET. Porém, as implicações e necessidade de investigação e tratamento da febre no pósoperatório de reconstruções laringotraqueais ainda não estão estabelecidas.

Continuaremos analisando, agora prospectivamente, os dados dos pacientes pediátricos submetidos a LTP e RCT no nosso Serviço, e continuaremos utilizando o balão de dilatação no tratamento da reestenose pós-reconstrução laringotraqueal.

Necessitamos mais estudos para determinar as causas das falhas terapêuticas e para aperfeiçoamento do manejo pós-operatório dessas crianças.

\section{CONCLUSÃO}

Apresentamos nossa série de 24 crianças com estenose laringotraqueal submetidas a reconstrução da via aérea em um único estágio. Nosso índice de decanulação total com LTP e RCT foi de 83,3\%.

\section{REFERÊNCIAS BIBLIOGRÁFICAS}

1. Koempel JA, Cotton RT. History of Pediatric Laryngotracheal Reconstruction. Otolaryngol Clin North Am. 2008, 41:825-35.

2. Cotton RT. Management of subglottic stenosis in infancy and childhood. Review of a consecutive series of cases managed by surgical reconstruction. Ann Otol Rhinol Laryngol. 1978, 87:649-57.

3. MyerCM III, O'Connor DM, Cotton RT. Proposed grading system for subglottic stenosis based on endotracheal tube sizes. Ann Otol Rhinol Laryngol. 1994, 103:319-23.

4. Boardman SJ, Albert DM. Single-Stage and Multistage Pediatric Laryngotracheal Reconstruction. Otolaryngol Clin North Am. 2008, 41:947-58.

5. Schroeder JW, Holinger LD. Congenital Laryngeal Stenosis. Otolaryngol Clin North Am. 2008, 41:865-75.

6. Gustafson LM, Hartley BEJ, Liu JH et al. Single-stage laryngotracheal reconstruction in children: A review of 200 cases. Otolaryngol Head Neck Surg. 2000, 123:430-4.

7. Sandu K, Monnier P. Cricotracheal Resection. Otolaryngol Clin N Am. 2008, 41:981-98.

8. Hartley BEJ, Cotton RT. Paediatric airway stenosis: laryngotracheal reconstruction or cricotracheal resection? Clin Otolaryngol. 2000, 25:342-9.

9. Schraff SA, Brumbaugh C, Meinzen-Derr J, Willging JP. The significance of post-operative fever following airway reconstruction. Int J Pediatr Otorhinolaringol. 2010, 74:5202.

10. Cotton RT. Management of laryngotracheal stenosis and tracheal lesions including single stage laryngotracheoplasty. Int J Ped Otorhinolaryngol. 1995, 32(Supplem):89-91.

11. Cotton RT, Myer CM III, O'Connor DM, et al. Pediatric laryngotracheal reconstruction with cartilage grafts and endotracheal tube stenting: the single-stage approach. Laryngoscope. 1995, 105:818-21. 